\title{
Modeling and Analysis of Ultrasonic Echoes Reflected from a Surface under Two Layers
}

\author{
Joyoni Dey and Takeo Kanade \\ Carnegie Mellon University
}

\begin{abstract}
Fat and muscle layers in the human soft-tissue causes degradation of resolution and distortions of shape in ultrasound images of organs. Novel ultrasonic applications such as registration between CT and ultrasound images, requiring comparable levels of accuracies between modalities could suffer from the distortions in ultrasound images. In this work, we describe a possible method to compensate or correct for these distortions in ultrasound images. This can be used as a pre-processing step before registration with other modalities. We derived a model of ultrasound field after reflection from an interface embedded in two layers. Experiments were performed using single-element transducers and custom made tissue mimicking phantoms of fat and muscle and a steel-block. We fit the model to the experimental data using Levenberg-Marquardt inversion.
\end{abstract}

Keywords: Medical ultrasound, biomedical modeling and simulation

\section{Introduction}

In addition to diagnostic applications, ultrasound is becoming increasingly popular for novel applications such as using intra-operatively obtained ultrasound images for registration with other modalities for computer-aided surgery. In Fig-

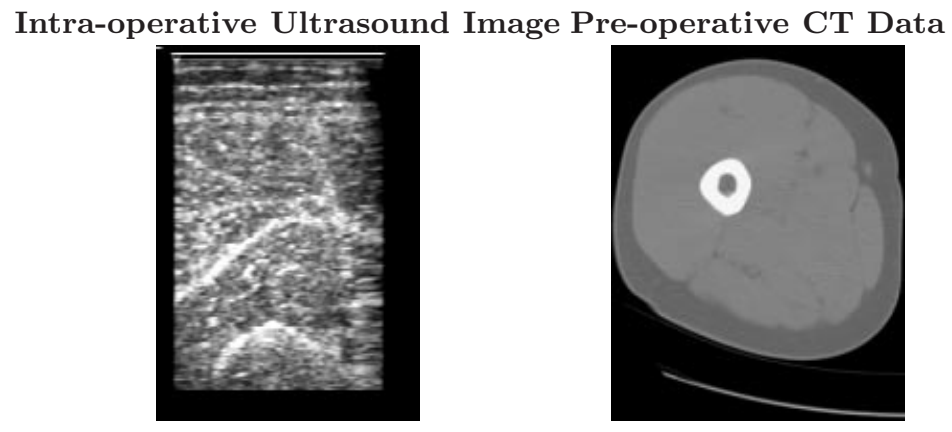

Fig. 1. Ultrasound image and CT slice of human femur 
ure 1 we show an ultrasound image of a human femur bone (the semi-circular reflection at the bottom) and a CT image of the femur. (Note that Figure 1 is shown for the purpose of illustration only - the images belong to different patients). It is evident that the bone outline can be used for the registration of the images. The problem with registration between these two modalities is that while CT-data is very accurate, ultrasound has imaging artifacts. In much of the human body there are layers of fat and muscle. These intervening layers can cause degradation of resolution, shape distortion and other artifacts.

We describe a method to compensate for these artifacts for the case of imaging the bone under fat and muscle layers. This method can be used as a preprocessing step to modify the ultrasound images before proceeding with the registration. We developed a forward ray-tracing model of the received ultrasound waves after propagation into two layers and reflecting back from a highly reflecting interface. We chose to model this particular set-up because it closely resembles the bone under layers of fat and muscle. Our derived model can handle arbitrary interface geometries and aperture configurations. We also describe an inversion process to estimate the parameters of the forward model. The main parameters of interest in our application are the depths of the interfaces for the case of two layers on a reflective interface.

We performed real-life experiment on uniform thickness tissue-mimicking phantoms with known thicknesses, placed on a steel-block. This set up mimics a fat and muscle layer on the highly reflective interface, the bone. We used a circular aperture transducer operating at $7.5 \mathrm{MHz}$. We then fit our forward model (for a special case where the layers are planar and parallel to the aperture) to this experimental data by iteratively updating the model parameters (the depths of the interfaces in this case) so as to minimize the least square-errors between the model and the data.

\section{Related Work}

Several authors have investigated the phase aberrations and amplitude distortions in ultrasound wave caused by layers of fat and muscle in the human body wall [1]- [11]. Some of them account for diffraction effects [1]- [4] while others consider ray-tracing approximations, which account for only reflection and refraction at the boundaries. When the organs of interest are large compared to the wavelength, ray-tracing can be used. Effects of composite layers on beamformation such as defocusing and beam-shifts and imaging artifacts such as splitimage, shadowing, and enhancements have been shown in the literature $[5,6, ?]$. In these works, ray-tracing has been used to explain these effects. While it does not consider diffraction, the advantage of ray-tracing based modeling is that it is highly parallelizable and fast. The cost of the implementation is particularly important if we are considering inversions of the model. It facilitates the inversion further if we have a closed form forward model. In a related work [12], we attempted to derive a fully analytical model for the propagation of a monochromatic spherical wave through general media geometries and arbitrary apertures 
under ray-tracing approximations. The modeling is similar to the work of Odegaard et al [10] - a main point of difference is that in the simulations described by Odegaard et al, the amplitude spreading factors were obtained numerically by perturbing rays in two directions, while we derived these factors in closed form by mathematically obtaining the ratios of differential flux-tube areas [12]. In this paper, when modeling the special case of imaging the bone under layers of fat and muscle, we used parts of our above mentioned results for propagation of the monochromatic spherical ultrasound wave through layered media.

\section{Theory: Forward Model}

In Figure 2(a), we show an ultrasonic aperture and a reflective interface (Plane 2) under two media. We have broken up the interface surfaces into small planar segments. The dimensions have to be of the order of the wavelength of the ultrasonic wave, about 0.2 to $0.3 \mathrm{~mm}$. We want to model the reflected waveform received at the aperture. First we consider a spherical wave emitted by a point in the aperture and observe the reflected waves for that source. The response for the entire aperture is the sum of the reflected waveforms received corresponding to each point source in the aperture. For a focussed aperture we can add the appropriate transmit and receive focusing delays before adding the responses from the different parts of the aperture. To derive our model, we used ray-tracing

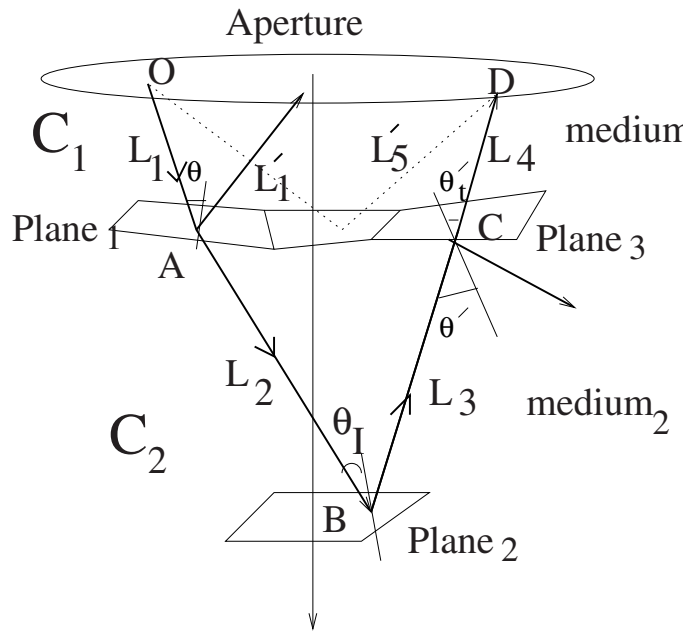

(a) Ray from source to receiver

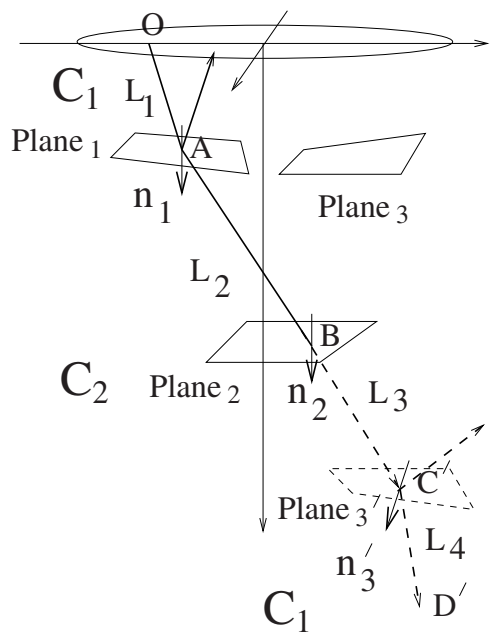

(b) Rays reflected about Plane 2

\section{Fig. 2. Ray Tracing from source to receiver}

assumption. Geometrically this implies that a spherical wave remains spherical after reflection. But refraction introduces a change in the shape of the waveform. Calculations show that for the parameters under consideration, the ray tracing 
approximation is not very restricting [12]. Other main assumptions are longitudinal propagation (valid for soft-tissues). We also considered absorption loss. Ignoring multiple reflections, the received wave at $D$ due to a point source at $O$, $f\left(P_{D}, t\right)$ would have two terms, $f_{1}\left(P_{D}, t\right)$ and $f_{2}\left(P_{D}, t\right)$. The first term, $f_{1}\left(P_{D}, t\right)$ is due to pure reflection from the interface between the first and second layer. The second term, $f_{2}\left(P_{D}, t\right)$ is the reflected wave from the second layer (it goes through refraction at the points $\mathrm{A}$ and $\mathrm{C}$ as well).

We can straightforwardly write the first term, for which the relevant ray-paths are those shown in dashed lines in Figure $2(\mathrm{a}) \cdot f_{1}\left(P_{D}, t\right)=\sum_{\text {rays' }} e^{-\left(\mu_{1}\left(L_{1}^{\prime}+L_{5}^{\prime}\right)\right)}$ $\frac{1}{\left(L_{1}^{\prime}+L_{5}^{\prime}\right)} V(\theta) \mathbf{p}\left[t-\left(\frac{\left(L_{1}^{\prime}+L_{5}^{\prime}\right)}{C_{1}}\right)\right]$ where inverse of the total path-length $L_{1}^{\prime}+L_{5}^{\prime}$ appears as the intrinsic attenuation factor (since this is pure reflection, the wave is spherical and for a spherical wave, the attenuation is $1 / r$, where $r$ is the equivalent radial distance covered from the source) ; $V(\theta)$ is the reflection coefficient, where $\theta$ is the angle of incidence of the ray. rays denotes the set of rays that reached from $O$ to $D$ on reflection at first interface. The exponent term is the term due to absorption loss. $\mu_{1}$ is the attenuation coefficient in the first medium.

The ray O-A-B-C-D, is the ray to be considered for the second term. This set is sufficient because of the linearity [12]. The segments OA and CD (with path lengths $L_{1}$ and $L_{4}$ ) are in the first medium with propagation velocity $C_{1}$. $\mathrm{AB}$ and $\mathrm{BC}$ (of lengths $L_{2}$ and $L_{3}$ ) are in the second medium, with propagation velocity $C_{2} . f_{2}\left(P_{D}, t\right)=\sum e^{-\left(\mu_{1} L_{1}+\mu_{2}\left(L_{2}+L_{3}\right)+\mu_{1} L_{4}\right)}\left|f_{D}\right| \mathbf{p}\left[t-\left(\frac{L_{1}}{C_{1}}+\frac{L_{2}+L_{3}}{C_{2}}+\frac{L_{4}}{C_{1}}\right)\right]$ where $L_{i}$ are the lengths of the path in each media for the ray (shown in solid lines) from each source to the point of interest in Figure 2(a). The first exponent is the absorption loss attenuation factor $[10,13,14] . \mu_{i}$ being the attenuation coefficients inside each medium. $\left|f_{D}\right|$ is the intrinsic attenuation factor, which is not exactly $1 / r$ anymore because of the refraction and so on.

It is an elaborate process to calculate this factor $\left|f_{D}\right|$, and is out of scope of this paper except for a brief outline. We can first simplify the rays - reflect ray $\mathrm{BC}$ and $\mathrm{CD}$ in Figure 2 (a) about the Plane 2, and imagine it as continuing along $\mathrm{AB}$ as shown in Figure 2(b). Now we see that as far as the derivation of $\left|f_{D}\right|$ is concerned, it is the same as if the wave was propagating into three-layers, except here the first and the third layers happen to be the same. In [12] we have formulated in detail the intrinsic attenuation factors after propagation through three (or more) media. We show the final result here, $\left|f_{D}\right|=R\left(\theta_{I}\right)|1+V(\theta)|\left|1+V^{\prime}\left(\theta^{\prime}\right)\right| \frac{1}{L_{1}} \sqrt{\frac{\Delta a a_{2} A_{1}}{\Delta D_{1} s_{1} S_{1}}} \sqrt{\frac{\cos \theta_{t}}{\cos \theta^{\prime}}}$. In this equation, $V(\theta)$ and $V\left(\theta^{\prime}\right)$ are the reflection coefficients at the first and the third interface. The angles are shown in Figure 2(a). The first factor $R\left(\theta_{I}\right)$ is a function of the incident angle $\theta_{I}$ corresponding to the reflection at the second interface (plane $B$ in Figure 2(b)). For a perfect reflector, this factor is unity. The area ratios $\sqrt{\frac{\Delta a a_{2} A_{1}}{\Delta D_{1} s_{1} S_{1}}}$ are the ratios of flux-tube areas that constructed perpendicular to the ray (part of the wavefront). Calculation of these areas in close-form are shown in [12]. Briefly, the approach taken is to change the ray differentially in two independent directions and calculate the sides of the triangular areas by taking 
derivative of the points of intersection of the rays and the interfaces. The areas of the triangles are then the magnitudes of cross-products of the sides.

The final received signal at point $D$ in the aperture due to the source at $O$ is the sum of $f\left(P_{D}, t\right)=f_{1}\left(P_{D}, t\right)+f_{2}\left(P_{D}, t\right)$. Finally, we can apply the aperture forward transmittance (for focusing, steering etc), if any, at each point and add the signals arriving at $D$ for all the point sources. Then to obtain the total response of the entire aperture, we would add the arriving wave at all the points in the aperture, appropriately modified by the received focusing delays, if any. For the unfocused transducer case, we would simply add the response arriving at all the points of the transducer, giving the final received time signal as

$$
\begin{aligned}
f(t) & =\sum_{P_{D}} \sum_{P_{O}} \sum_{\text {rays }^{\prime}} e^{-\left(\mu_{1}\left(L_{1}^{\prime}+L_{5}^{\prime}\right)\right)} \frac{1}{\left(L_{1}^{\prime}+L_{5}^{\prime}\right)} V(\theta) \mathbf{p}\left[t-\left(\frac{\left(L_{1}^{\prime}+L_{5}^{\prime}\right)}{C_{1}}\right)\right] \\
& \left.+\sum_{P_{D}} \sum_{P_{O}} \sum_{\text {rays }} e^{-\left(\mu_{1} L_{1}+\mu_{2}\left(L_{2}+L_{3}\right)+\mu_{1} L_{4}\right)}\left|f_{D}\right| \mathbf{p}\left[t-\left(\frac{L_{1}}{C_{1}}+\frac{L_{2}+L_{3}}{C_{2}}+\frac{L_{4}}{C_{1}}\right)\right] 1\right)
\end{aligned}
$$

where the integral over $P_{O}$ indicates we sum the waves arrived at $D$ for all the point sources, the integral over $P_{D}$ indicates that we then add the received waveform at each such point in the aperture.

\section{Inversion of the Model}

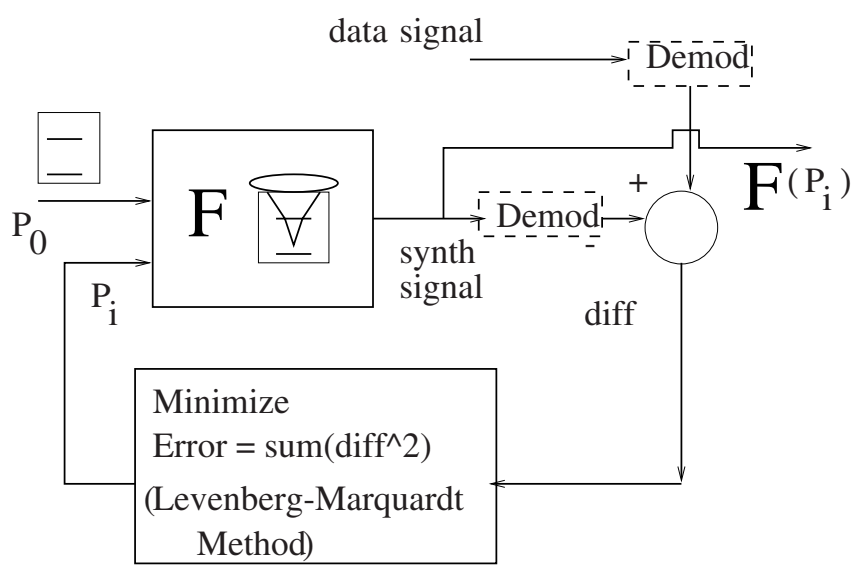

Fig. 3. Iterative Inversion

We wrote a simulator to obtain a synthetic output of the received signal given in Equation 1, given the input parameters of the receiver, and the media parameters - the interface geometries and the physical variables. This is the forward model, denoted by block diagram $F$ in Figure 3. We use this block 
diagram to show the inversion of the model (ignoring the dotted blocks for the present). We start with an initial estimate of the parameter values, $P_{0}$ (in this case the depth of the two interfaces from the aperture). The initial estimate is chosen by a simple thresholding operation on the experimental data signal. The parameters are used by the forward model $F$ to get a synthesized signal output (or an ultrasound image in general). The error difference between the synthesized output and the actual experimental data signal (or images) drives the parameters to their next values. We adopt the Levenberg-Marquardt minimization for this purpose. The simulated output signal from the receiver in our case is a time signal, $y=y(n ; \mathbf{p})$ where $n$ is the time sample index and $\mathbf{p}$ is the parameter set used. In this case, the parameter set consists of the two depths of the planar interfaces, given by $D_{1}$ and $D_{2}$. The scalar error that is minimized is given by $E(\mathbf{p})=\sum_{n}\left(y_{n}-y(n ; \mathbf{p})\right)^{2}$ where $y_{n}$ and $y(n ; \mathbf{p})$ are the data signal and the synthesized signal respectively.

\section{Experiments and Model Fitting}

\subsection{Experimental Equipment and Procedure}

The ultrasonic transducer used is a $7.5 \mathrm{MHz}$, single element circular aperture transducer. We use a RiTec control box with variable amplitude and frequency to trigger the transducer. We excite the transducer with a short square pulse of time-duration half of the time-period at resonance, (about 67 nano-secs). We place the transducer over the fat-phantom and obtain the return-echoes. The echoes are captured, and then filtered by an anti-aliasing low pass (cut off 10 $\mathrm{MHz}$ ) and a high pass filter (to remove the DC bias, cut off at $20 \mathrm{KHz}$ ). The filtered signal is sampled at $25 \mathrm{MHz}$ by an Sonix analog to digital converter. The Sonix board is triggered by a synchronizing trigger source from the control box. We use "fat" and "muscle" mimicking phantoms. These physical objects designed from water-agar-gel mixtures mimic some ultrasound characteristics (such as propagation velocity) in human fat and muscle. We also use a steelblock about $5 \mathrm{~cm}$ thick. To create a highly reflective interface under the two layers (fat and muscle) the phantoms are stacked on the steel-block so that the muscle-phantom lies on the steel-block and the fat-phantom lies on top of the muscle. To obtain good coupling between the phantom layers, they are wetted with water.

In order to invert the forward model $F$ in Equation 1, we need to determine the pulse $\mathbf{p}(t)$ for this transducer. We do so this experimentally by a standard method of obtaining an echo from a steel-block under-water. The pulse obtained is observed to be nearly a Gaussian. We fit a Gaussian curve (in amplitude, mean, standard deviation, and the carrier frequency) using the simplex algorithm. We obtain the pulse to within a scale. The phantom parameters such as $m u_{i}, C_{i}, \rho_{i}$ are provided by the manufacturer. 
Table 1. Convergence Data

\begin{tabular}{||l|l|l||l|l|l||}
\hline \hline \multicolumn{2}{||c||}{ Using Demodulated Signals } & \multicolumn{3}{|c||}{ Using Modulated Signals } \\
\hline & Start & End & & Start & End \\
\hline Error & 45306 & 6203 & Error & 32651 & 12702 \\
\hline Depth $D_{1}$ & $15.0000 \mathrm{~mm}$ & $14.9568 \mathrm{~mm}$ & Depth $D_{1}$ & $14.9568 \mathrm{~mm}$ & $14.9727 \mathrm{~mm}$ \\
\hline Depth $D_{2}$ & $35.3000 \mathrm{~mm}$ & $35.4342 \mathrm{~mm}$ & Depth $D_{2}$ & $35.4342 \mathrm{~mm}$ & $35.4220 \mathrm{~mm}$ \\
\hline
\end{tabular}

\subsection{Results of Inversion}

A straightforward inversion of the forward model has the problem that, due to the modulation of the signals at the carrier frequency, $f_{0}$ there would be local minima at regular intervals of roughly $\lambda_{0}$ from the global minimum [12]. To obtain a smoother error bowl, we first need to add a demodulator shown in dotted lines in Figure 3, so that the envelopes of the signals should be involved in the minimization, rather than the modulated signals themselves. The signals are real and typically the energy is concentrated (in "humps") around the carrier $f_{0}$. There are many techniques to demodulate signals such as using the Hilbert Transform. We adopted simple intuitive steps described briefly as follows. The receiver signals are real, hence the real part of their FFT is symmetric and the imaginary part, antisymmetric. All the information of the signal is then entirely available on one side of the spectrum. We extract the real and imaginary "humps" of the spectrum from the positive side of frequency (humps centered around the positive $f_{0}$ ). We shift each of them by $f_{0}$ (to baseband) to get $G_{r}(f)$ and $G_{i}(f)$. The signal $g(n)=\operatorname{IFFT}\left(G_{r}(f)+j G_{i}(f)\right)$ therefore has all the information of the original signal and is a baseband signal. But $g(n)$ is complex [12] and difficult to use in the minimization as such. Hence we use the real function $s_{n}=\sqrt{2 g(n) g^{*}(n)}$. The $\sqrt{2}$ factor is needed such that the original modulated signal and the baseband "envelope" signal $s_{n}$ has the same energy. Some examples are given in [12].

After convergence with the envelope signals, we are roughly within $\lambda / 2$ of the global minimum, we revert back to using the raw (modulated RF) signals again (increased sensitivity) and minimize the difference between those to do fine-tuning of the parameter fitting.

From a rough estimate of the location of the echoes from the experimental "data" signal, we start the iteration at $D_{1}=15.0 \mathrm{~mm}$ and $D_{2}=35.3 \mathrm{~mm}$. The starting and final errors and converged parameters are given in the second and third columns of Table 1. After this stage the echoes seem to be slightly out of phase (particularly the first one). This is because so far we used the envelope of the signals for the error minimization rather than using the signals themselves. Hence, the next obvious fine-tuning step is to eliminate the demodulation step and run the inversion on the raw signals. The error and parameter values are shown in the last three columns in Table 1. The starting parameters for these sets of iterations (using the raw signals) are the end parameters arrived at by using the demodulated signals (third column, Table 1. We observe that for the 
same parameters, the starting error is much larger for the modulated case than for the demodulated case in Table 1. And the final error is higher than what we obtained at the end of the runs for the demodulated case. This is not an unexpected result. Matching of envelopes is a different criterion from matching of the signals themselves.

As a last step, we do the scale correction between simulation and experiment. We assume that the initial estimate of the scale (by energy ratio of the signals) was near enough to the correct scale so as not to hamper the convergence of the other parameters. Once we obtain a good estimate of the parameters $\mathbf{p}=\left[D_{1}, D_{2}\right]$, we can correct for the scale factor analytically. At the correct parameters, $\mathbf{p}_{\mathbf{c}}$, the energy is given by $E(\mathbf{p})=\sum_{n}\left(y_{n}-A y\left(n ; \mathbf{p}_{\mathbf{c}}\right)\right)^{2}$. The right scale, at which this error is minimum, can be calculated by setting the derivative of $E(\mathbf{p})$ with respect to $A$ to zero. This is given by $A_{c}=\frac{\sum_{n} s_{n} s\left(n ; \mathbf{p}_{\mathbf{c}}\right)}{\sum_{n} s^{2}\left(n ; \mathbf{p}_{\mathbf{c}}\right)}$. The original scale was $A=4.4904$. After the correction, the scale is $A_{c}=3.9139$. The correctly-scaled simulation and the experiments are shown in Figure 4.

Figure 4 show a qualitatively good match between experiments and simulations. The first echo seems to have matched well except toward end of the pulse. The second echo seems to have matched in shape well in the midsection but not in the beginning and the end. We note that we have not modeled the noise in our simulations. When the signal is small (towards the beginning/end of the echoes) the effect of the noise is higher. We calculate some quantitative ratios between the compared experimental and simulation echoes shown in Figure 4. Considering the first echo, the RMS amplitude ratio of the experimental one to that of the the fitted model is close to unity, 1.0353 (or $0.1507 \mathrm{db}$ ). For the second echo, the RMS amplitude ratio of the experimental to that of the fitted model is 0.9564 (or $-0.1936 \mathrm{db}$ ). The final parameters are obtained as $D_{1}=14.9727$ and $D_{2}=35.4220$. Hence the layer thicknesses are within $0.03 \mathrm{~mm}$ and $.45 \mathrm{~mm}$ of the manufacturer specified nominal values of $15 \mathrm{~mm}$ and $20 \mathrm{~mm}$.

\section{Conclusions}

In this work, we have modeled ultrasound echo-field reflected from a interface embedded in two layers. We performed experiments on tissue mimicking phantoms and fit our model to the data. For model-fitting, we have minimized the mean-squared-error using the Levenberg-Marquardt minimization technique. We obtained good quantitative matches.

It is noted that ultrasound speckle has not be considered here in simulations or experiments. The speckle is expected to add noise to the error function to be minimized, thereby making the inversion more prone to false minima. The effect can be reduced to some extent perhaps by using multiresolution approach - first using smoothing functions on the envelopes and obtaining a minima roughly close to the global minimum ; and then using the end parameters of that step as the initial guess into the next resolution step; and so on until finally using the original signals to fine tune the minima location. 

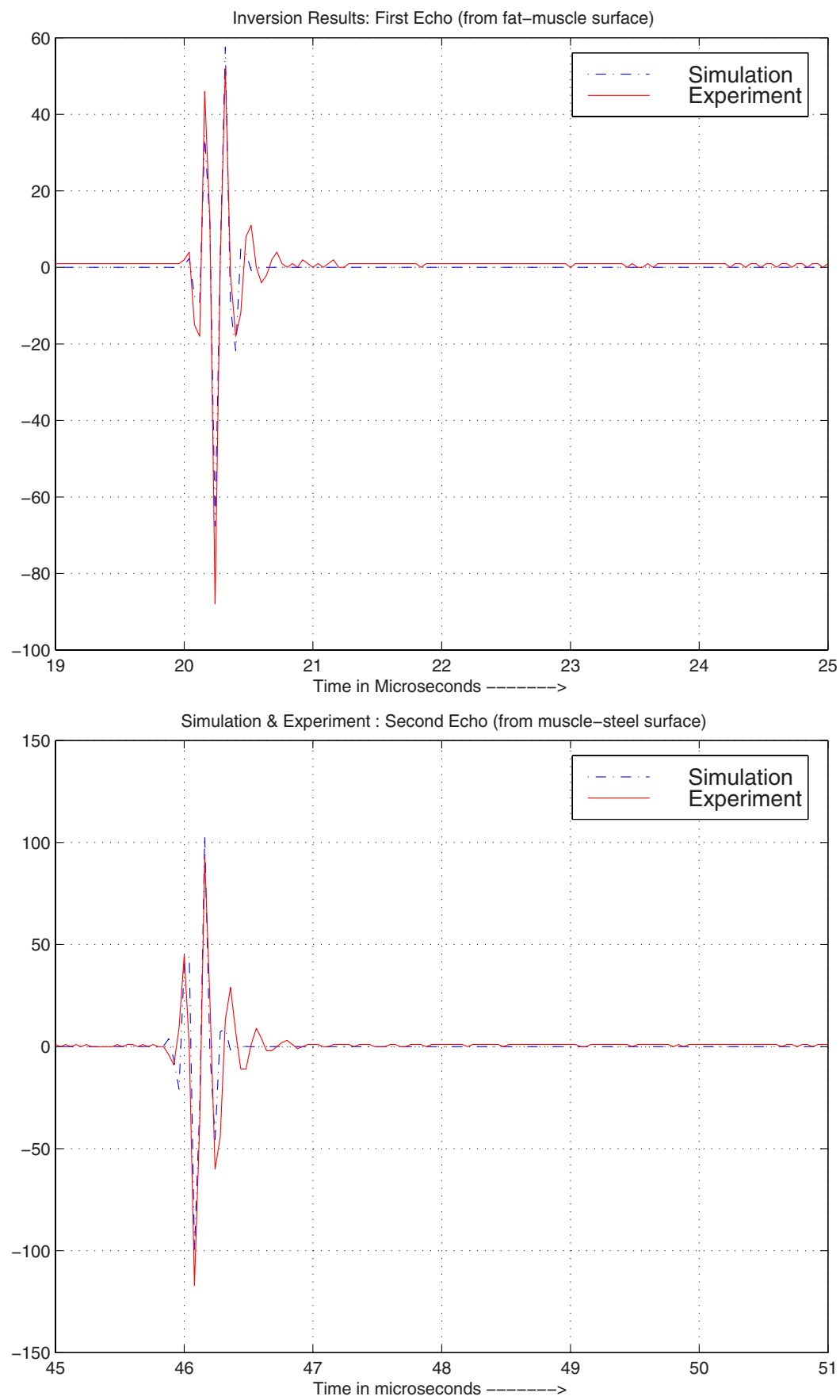

Fig. 4. Comparison of Experiment and Simulation: first (top) and second (bottom) echo 
Finally, it is noted even though we presented results for a single-element ultrasound transducer, the method is not fundamentally different for a linear array aperture. Each rectangular element of the array can be first discretized to point sources emitting spherical waves. Distortion of each emitted wave due to layered inhomogeneity can be calculated as shown here. Then transmit/receive focusing delays can be applied to the rectangular elements of the array and the result summed to obtain each of the scan lines.

Acknowledgments: We would like to thank Rajesh Gopakumar and Farhana Kagalwala for many helpful discussions and suggestions.

\section{References}

1. T. D. Mast, L. M. Hinkelman, L. A. Metley, M. J. Orr, R. C. Waag, "Simulation of ultrasonic pulse propagation, distortion, and attenuation in the human chest wall", J. Acoust. Soc. Am. 106, 3665-3677, December 1999

2. L. M. Hinkelman, T. D. Mast, L. A. Metley, and R. C. Waag, "The effect of abdominal wall morphology on ultrasonic pulse distortion. Part I: Measurements", J. Acoust. Soc. Am. 104 3635-3649 (1998)

3. T. D. Mast, L. M. Hinkelman, M. J. Orr, and R. C. Waag, "The effect of abdominal wall morphology on ultrasonic pulse distortion. Part II: Simulations", J. Acoust. Soc. Am. 104 3651-3664 (1998)

4. C. W. Mantry and S. L. Broschat, "FDTD Simulations for Ultrasound Propagation in a 2-D Breast Model", Ultrason. Imaging 18 25-34 (1996)

5. G. Kossoff, D.A. Carpenter, D.E. Robinson, D. Ostry and P.L. Ho, "A sonographic technique to reduce beam distortion by curved transducers," Ultrasound in Med. and Biol. 15, 375-382(1989).

6. D. E. Robinson, L. S. Wilson, and G. Kossoff, "Shadowing and enhancement in ultrasonic echograms by reflection and refraction", J. Clin. Ultrasound, 9, 181-188 (1981)

7. E. E. Sauerbrei, "The split image artifact in pelvic ultrasonography: the anatomy and physics," J. Ultrasound Med. 4, 29-34 (1985)

8. D. A. Carpenter, D. E. Robinson, P. L. Ho, D.C.C. Martin and P. Isaacs, "Body wall aberration correction in medical ultrasonic images using synthetic-aperture data," IEEE 1993 Ultrason. Symp. Proc. 3, 1131-1134(1993).

9. D.A. Carpenter, G. Kossoff and K. A. Griffiths, "Correction of distortion in US images caused by subcutaneous tissues: Results in tissue phantoms and human subjects," Radiology 195 563-567(1995).

10. L. Odegaard, S. Holm, F. Teigen and T. Kleveland, "Acoustic field simulation for arbitrarily shaped transducers in a stratified medium," IEEE 1994 Ultrason. Symp. Proc. 1535-1538 (1994).

11. L. Odegaard, S. Holm and H. Torp, "Phase aberration correction applied to annular array transducers when focusing through a stratified medium," IEEE 1993 Ultrason. Symp. Proc. 1159-1162(1993).

12. J. Dey, Modeling and analysis of ultrasound propagation in layered medium. Doctoral Dissertation, Department of ECE, Carnegie Mellon University, 1999, CMURI-TR-99-26.

13. L. M. Brekhovskikh, Waves in layered media (Academic Press, 1980).

14. A. D. Pierce, Acoustics: An introduction to its physical principles and applications (McGraw Hill, Inc, 1981). 